ANNALS OF

CLINICAL

NEUROPHYSIOLOGY

\title{
Pattern analysis of lower limb magnetic resonance images in Korean patients with distal myopathy
}

\author{
Hyung Jun Park ${ }^{1,2}$, Ha Young Shin ${ }^{2}$, Seung Min Kim², Kee Duk Park ${ }^{3}$, and Young-Chul Choi ${ }^{2}$ \\ ${ }^{1}$ Department of Neurology, Gangneung Asan Hospital, University of Ulsan College of Medicine, Gangneung, Korea \\ 2Department of Neurology, Yonsei University College of Medicine, Seoul, Korea \\ ${ }^{3}$ Department of Neurology, Mokdong Hospital, Ewha Womans University School of Medicine, Seoul, Korea
}

Received: June 5, 2018

Revised: July 10, 2018

Accepted: July 18, 2018

\section{Correspondence to}

Young-Chul Choi

Department of Neurology, Gangnam Severance Hospital, Yonsei University College of

Medicine, 211 Eonju-ro, Gangnam-gu, Seoul 06273, Korea

Tel: +82-2-2019-3323

Fax: +82-2-3462-5904

E-mail:ycchoi@yuhs.ac

ORCID

\section{Hyung Jun Park}

https://orcid.org/0000-0003-4165-8901

Ha Young Shin

https://orcid.org/0000-0002-4408-8265

\section{Seung Min Kim}

https://orcid.org/0000-0002-4384-9640

Kee Duk Park

https://orcid.org/0000-0003-1628-6683

\section{Young-Chul Choi}

https://orcid.org/0000-0001-5525-6861

Background: Magnetic resonance (MR) images are useful for diagnosing myopathy. The purpose of this study was to determine the usefulness of lower-limb MR images in Korean patients with distal myopathy.

Methods: We reviewed medical records in the myopathy database from January 2002 to October 2016. We selected 21 patients from 91 unrelated families with distal myopathy: four with GNE myopathy, 11 with dysferlinopathy, and six with ADSSL1 myopathy.

Results: Ten (48\%) of the 21 patients were men. The ages of the participants at symptom onset and imaging were $19.2 \pm 9.5$ and $30.4 \pm 9.0$ years (mean \pm standard deviation), respectively. Their grade on the modified Gardner-Medwin and Walton grade was $3.3 \pm 1.7$. The strength grade of the knee extensors was not correlated with the Mercuri scale for the quadriceps $(r=-0.247, p=0.115)$. However, the Medical Research Council grades of the knee flexors, ankle dorsiflexors, and ankle plantar flexors were significantly correlated with the Mercuri scale ratings of the knee flexors ( $r=-0.497, p=0.001)$, tibialis anterior ( $r=-0.727, p<0.001)$, and ankle plantar flexors ( $r=-0.620, p<0.001)$, respectively. T1-weighted MR images showed characteristic fatty replacement patterns that were consistent with the causative genes. Unsupervised hierarchical clustering of the Mercuri scale showed that the main factors contributing to the dichotomy were the causative gene and the clinical severity.

Conclusions: This study is the first to reveal the usefulness of lower-limb MR images in the differential diagnosis of distal myopathy in Korea.

Key words: Distal myopathies; Magnetic resonance imaging; DYSF; GNE; ADSSL1 


\section{INTRODUCTION}

Distal myopathy is a clinically and genetically heterogeneous group of hereditary muscle disorders characterized by the progressive weakness and atrophy of distal muscles. Thus far, 16 causative genes have been reported in distal myopathy: DYSF, TTN, GNE, MYH7, MATR3, TIA1, MYOT, NEB, CAV3, LDB3, ANO5, DNM2, KLHL9, FLNC, VCP, and ADSSL1.' Mutations in DYSF, GNE, and ADSSL1 are the most common causes of distal myopathy in Korea. ${ }^{2}$

Muscle magnetic resonance (MR) images are useful for diagnosing myopathy. ${ }^{3} \mathrm{~T} 1$-weighted images can be used to assess the fatty replacement of muscle and the topography of neuromuscular lesions. ${ }^{4}$ T2-weighted images or short tau inversion recovery (STIR) images help when assessing muscle edema, necrosis, or inflammation occurring in a myopathy condition. ${ }^{4}$ Muscle MR images are particularly useful for identifying targets of muscle biopsies, patterns of muscle involvement, and patients with subclinical myopathy. Moreover, since MR imaging is a noninvasive technique and does not use ionizing radiation, muscle MR imaging provides an excellent approach for measuring the progression of muscle disease in clinical trials. ${ }^{5}$ However, it is well known that the pattern analysis of muscle MR images is sensitive but not specific, and few studies have investigated the pattern analysis of myopathy in Korean populations. ${ }^{2}$ To further clarify the usefulness of lower-limb MR images in Korean patients with distal myopathy, we analyzed the radiological patterns of three common types of distal myopathy: GNE myopathy, dysferlinopathy, and ADSSLI myopathy.

\section{MATERIALS AND METHODS}

\section{Study participants}

We reviewed medical records in the myopathy database from January 2002 to October 2016. The study hospital is one of the largest myology centers in Korea and the database contained 91 unrelated families with distal myopathy. The 21 patients examined comprised four unrelated patients with GNE myopathy, 11 unrelated patients with dysferlinopathy, and six unrelated patients with ADSSL 1 myopathy. Twenty patients were confirmed genetically, and one patient (II-2 in MF1152) was diagnosed by the absence of dysferlin staining in immunohistochemistry. All patients cooperated fully and provided written consents for their inclusion in both the genetic and imaging examinations. Our Institutional Review Board approved the research protocol (IRB No: 3-2016-0283).

\section{Phenotype assessment}

The clinical information used in the phenotype assessment included sex, age at symptom onset, muscle impairments, and deep tendon reflexes. The powers of knee extension, knee flexion, ankle dorsiflexion, and ankle plantar flexion were categorized using the Medical Research Council (MRC) grading system. Physical disability was evaluated using nine grades of the modified Gardner-Medwin and Walton (GMW) grade as described previously: ${ }^{6}$ grade $0=$ hyperCKemia, with all activities normal; grade $1=$ normal gait, unable to run freely, and myalgia; grade 2 = unable to walk on tiptoes, and waddling gait; grade 3 = evident muscular weakness, steppage gait, and only able to climb stairs with a banister; grade $4=$ difficulty rising from the floor, and Gowers' sign; grade $5=$ unable to rise from the floor; grade $6=$ unable to climb stairs; grade $7=$ unable to rise from a chair; grade $8=$ unable to walk unassisted; and grade $9=$ unable to eat, drink, or sit without assistance.

\section{Magnetic resonance imaging of muscles}

Lower-limb MR images of the thigh and calf muscles were acquired using a 1.5-T system (MAGNETOM Avanto, Siemens Healthcare, Erlangen, Germany). T1-weighted imaging was performed in the axial plane (field of view $[\mathrm{FOV}]=24 \times 32 \mathrm{~cm}$, slice thickness $=10 \mathrm{~mm}$, and slice gap $=1 \mathrm{~mm}$ ) and the coronal plane $(F O V=50 \times 32 \mathrm{~cm}$, slice thickness $=5-6 \mathrm{~mm}$, and slice gap $=2 \mathrm{~mm}$ ). T1-weighted turbo spin-echo (TSE) imaging was performed with the following parameters: repetition time (TR)/echo time $(\mathrm{TE})=445-730 / 9.5-11 \mathrm{~ms}$, echo train length $=3-5$, and matrixes with 320-384 $\times$ 192-269 pixels. To assess muscle edema or inflammation, fat-suppressed T2-weighted TSE imaging (TR/TE = 3,000$3,200 / 85-90 \mathrm{~ms}$, echo train length $=13$, and matrix with $512 \times 512$ pixels) was performed with the same FOV, slice thickness, and slice gap as in T1-weighted imaging.

The degree of muscle degeneration was evaluated by applying the Mercuri scale to T1-weighted MR images as follows: ${ }^{7,8} 0$ points $=$ normal appearance; 1 point $($ mild $)=$ 
traces of increased signal intensity; 2 points (moderate) $=$ increased signal intensity with confluence in less than $50 \%$ of the muscle; 3 points (severe) = increased signal intensity in more than $50 \%$ of the muscle; and 4 points (end-stage disease) $=$ increased signal intensity over the entire muscle.

\section{Statistical analysis}

Correlations between the Mercuri scale and MRC grade were evaluated with the Spearman method for the knee extensors, knee flexors, ankle dorsiflexors, and ankle plantar flexors. Differences were considered statistically signifcant at $p \leq 0.05$. All statistical analyses were conducted using R software (version 3.1.2, www.r-project.org). Heat maps and hierarchical clustering were generated with the gplots package of R software.

\section{RESULTS}

\section{Clinical presentation}

The clinical phenotypes of the 21 patients with distal myopathy are summarized in Table 1. Ten (48\%) of all patients were men. The ages of the participants at symptom onset and imaging were $19.2 \pm 9.5$ and $30.4 \pm 9.0$ years (mean \pm standard deviation), respectively. The grade on the modified GMW grade was $3.3 \pm 1.7$. The muscle strengths of the knee extensors, knee flexors, ankle dorsiflexors, and ankle plantar flexors were $4.42 \pm 0.55,4.23 \pm 1.03,3.61 \pm 1.36$, and $3.94 \pm$ 0.59 , respectively (Fig. 1).

The participants showed a variety of clinical manifestations that were related to their specific causative genes. Four patients with GNE myopathy showed predominant motor weakness of the ankle dorsiflexors, with relative sparing of the knee extensors. All 11 patients with dysferlinopathy showed predominant motor weakness of the ankle plantar flexors. Five of the six patients with ADSSL1 myopathy (II-2 in MF1191, II-2 in MF1004, II-1 in MF578, II-2 in MF416, and I-2 in MF795) showed predominant weakness of the ankle dorsiflexors, and three patients (II-2 in MF1004, II-2 in MF416, and I-2 in MF795) also had motor weakness of the quadriceps. Patient II-2 in MF1184 showed diffuse muscle weakness, but she had predominant weakness of the ankle dorsiflexors approximately one year after the diagnosis and in MR images.

\section{Radiological analysis}

We evaluated whether the degree of muscle fatty replacement observed in lower-limb MR images was correlated with muscle strength by comparing the MRC grade and Mercuri scale in the quadriceps (rectus femoris, vastus lateralis, vastus medialis, and vastus intermedius), knee flexors (semitendinosus, semimembranosus, and the short and long heads of biceps femoris), tibialis anterior, and ankle plantar flexors (soleus and medial/lateral gastrocnemius). The MRC grade of the knee extensors was not correlated with the Mercuri scale rating of the quadriceps $(r=-0.247, p=0.115)$. However, the MRC grades of the knee flexors, ankle dorsiflexors, and ankle plantar flexors were significantly correlated with the Mercuri scale ratings of the knee flexors $(r=-0.497, p=0.001)$, tibialis anterior $(r=-0.727, p<0.001)$, and ankle plantar flexors ( $r=$ $-0.620, p<0.001)$, respectively.

T1-weighted MR images showed characteristic fatty replacement patterns depending on the specific causative genes (Fig. 2). Firstly, the short head of the biceps femoris was mainly affected in four patients with GNE myopathy, followed by the adductor longus and semitendinosus at the thigh level. The vastus lateralis was the only muscle spared in advanced stages. At the calf level, the tibialis anterior and extensor digitorum longus were mainly affected, followed by the soleus, peroneus longus, and tibialis posterior (Supplementary Fig. 1). Secondly, the 11 patients with dysferlinopathy exhibited fatty replacement that generally began in the semimembranosus and then affected the adductor magnus and vastus lateralis. However, the rectus femoris, gracilis, and sartorius were relatively spared. At the calf level, the fatty replacement generally began in the medial gastrocnemius and then affected the lateral gastrocnemius and soleus (Supplementary Fig. 2). Thirdly, the six patients with ADSSL1 mutations showed predominant fatty replacement of the biceps femoris and vastus lateralis at the thigh level. At the calf level, fatty replacements of the gastrocnemius began at the early stages (Supplementary Fig. 3). Unsupervised hierarchical clustering of the Mercuri scale ratings showed that the included patients could be divided into two distinct subpopulations: one with mild fatty replacement and the other with moderate/severe fatty replacement (Fig. 3). The main factors contributing to this dichotomy were the causative gene and the clinical severity (as assessed using the modified GMW grade). STIR sequences showed fre- 


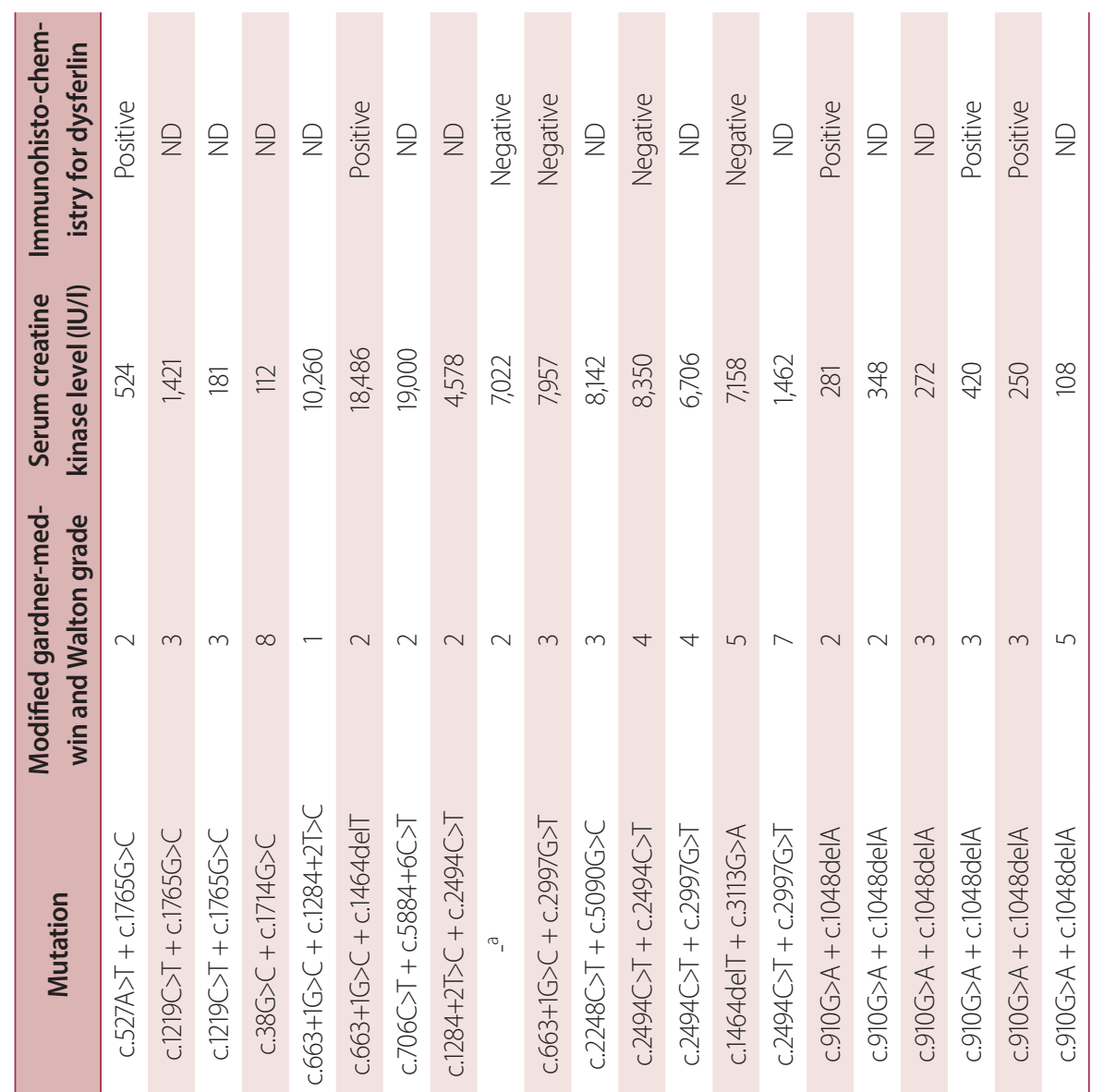

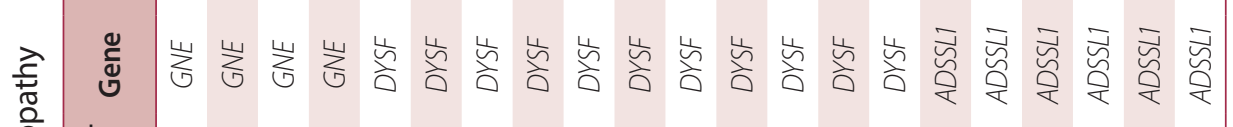


A

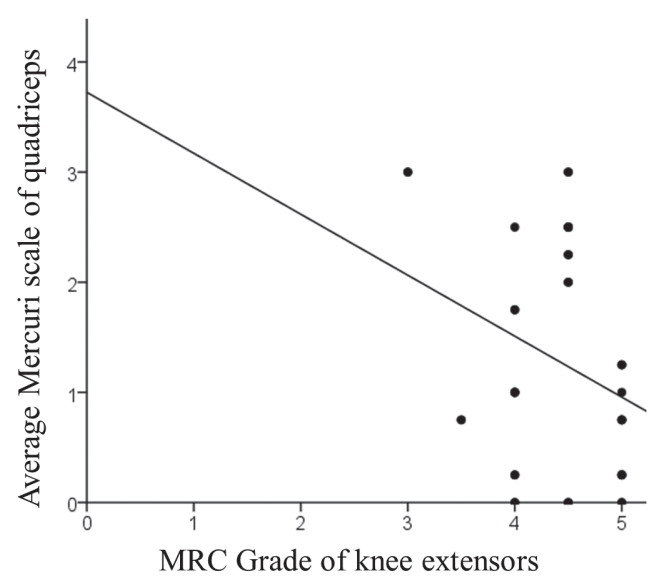

C

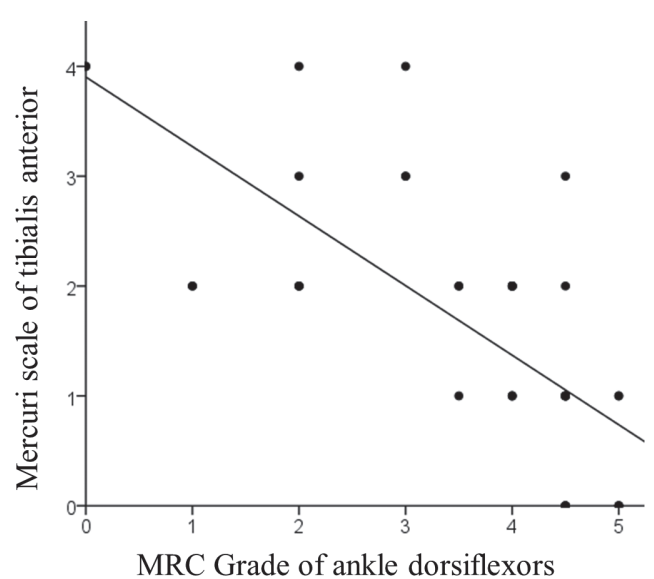

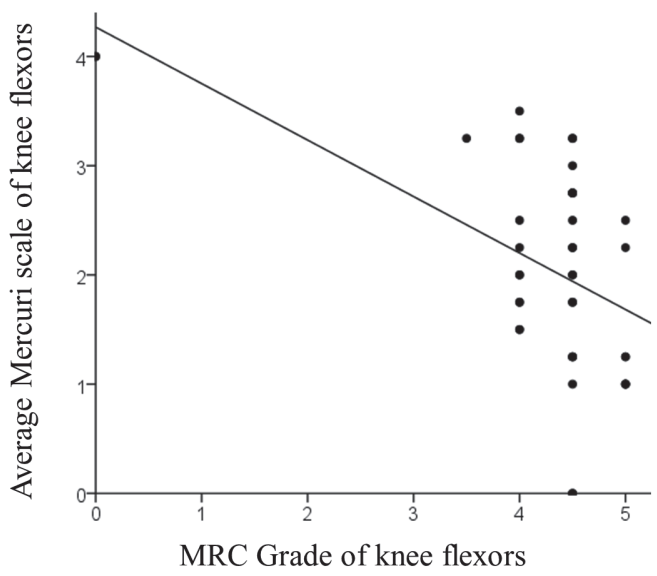

B

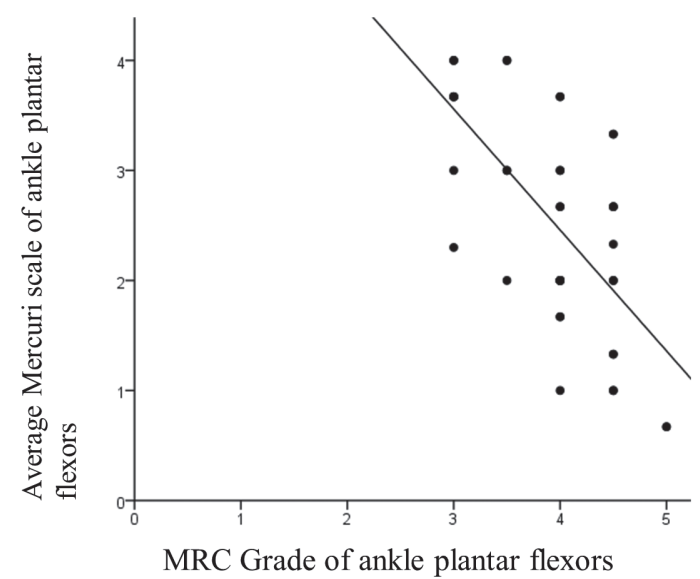

Fig. 1. Relationship between muscle strength and the degree of fatty replacement observed in lower-limb magnetic resonance (MR) images. (A) The Mercuri scale rating of the quadriceps (rectus femoris, vastus lateralis, vastus medialis, and vastus intermedius) was not significantly correlated with the Medical Research Council (MRC) grade of the knee extensors ( $r=-0.247, p=0.115$ ). (B) The Mercuri scale rating of the knee flexors (semitendinosus, semimembranosus, and the short and long heads of biceps femoris) was significantly correlated with the MRC grade of the knee flexors ( $r=-0.497$, $p=0.001$ ). (C) The Mercuri scale rating of the tibialis anterior was significantly correlated with the MRC grade of the ankle dorsiflexors $(r=-0.727, p<0.001)$. (D) The Mercuri scale rating of the ankle plantar flexors (soleus and medial/lateral gastrocnemius) was significantly correlated with the MRC grade of the ankle plantar flexors $(r=-0.620, p<0.001)$.

quent edema in the adductor magnus and gastrocnemius in the patients with dysferlinopathy.

\section{DISCUSSION}

The present study has revealed distinct fatty replacement patterns in lower-limb MR images of Korean patients with different genotypes of distal myopathy. However, unsupervised hierarchical clustering of the Mercuri scale was unable to separate the patients into three groups consistent with their genotypes.

The clinical manifestations of the present patients were consistent with those reported previously for GNE myopathy, dysferlinopathy, and ADSSL1 myopathy. Patients with GNE myopathy showed selective involvement of the tibialis anterior and mild creatine kinase (CK) elevation, with relative sparing of the quadriceps. ${ }^{9,10}$ Patients with dysferlinopathy showed adult-onset, predominant ankle plantar flexor weakness, no facial muscle involvement, and marked CK elevation. ${ }^{11,12}$ Patients with ADSSL1 myopathy showed diffuse muscle weakness initially, followed by distal muscle weak- 


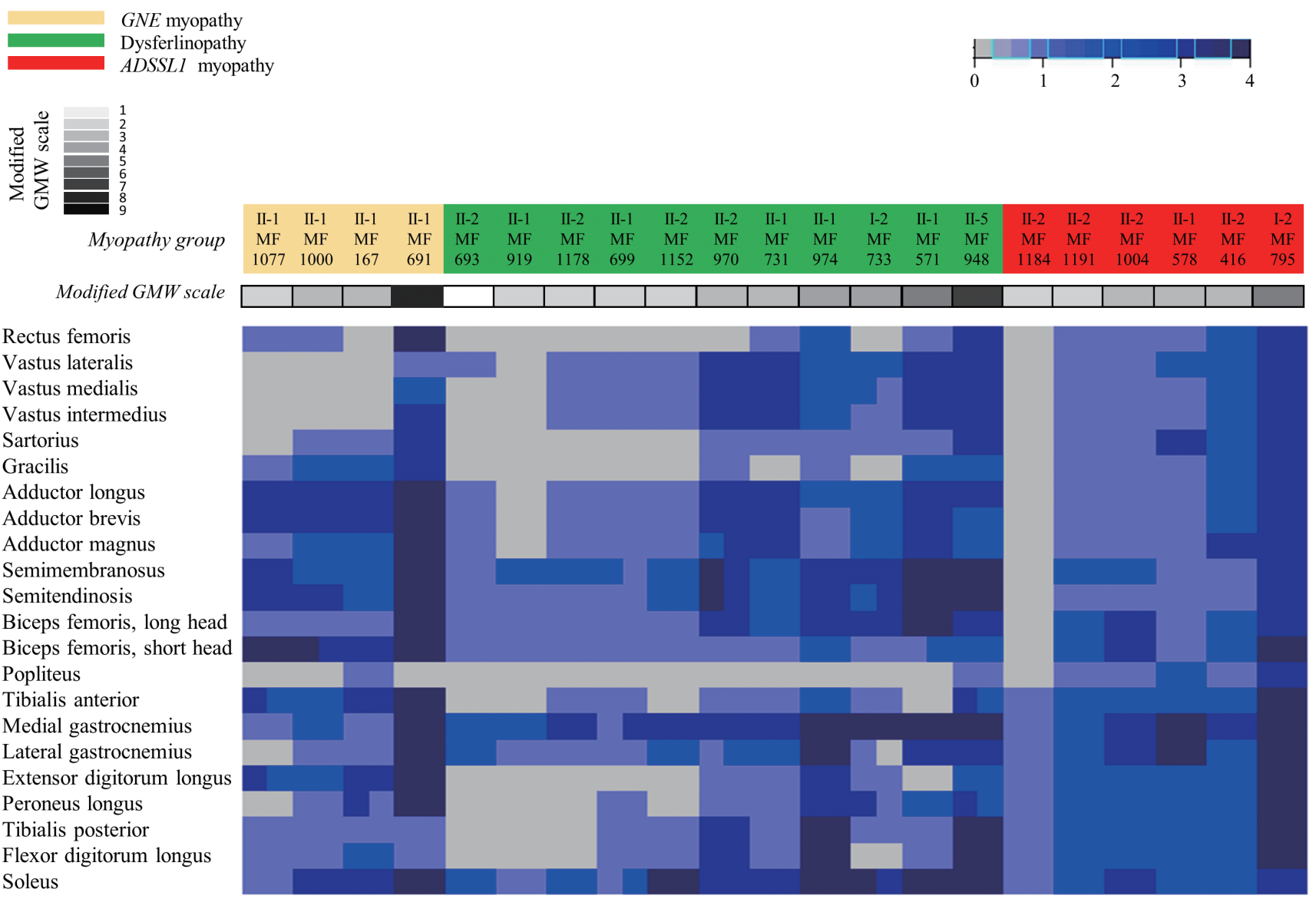

Fig. 2. Heat map representing muscle degeneration. GNE myopathy was characterized by the early involvement of the tibialis anterior and the short head of the biceps femoris, with relative sparing of the vastus lateralis. Dysferlinopathy showed the early involvement of the posterior compartment of the calf and thigh muscles, with relative sparing of the sartorius, gracilis, and rectus femoris. ADSSL1 myopathy showed fatty replacements of the gastrocnemius that began at the early stages. Each column in the heat map corresponds to one patient. Each row corresponds to one muscle, in descending order from cranial to caudal. A gray-blue-midnight-blue gradient in the heat map indicates increasing fatty substitution (legend at the top). The two rows at the left of the heat map denote individual clinical features: the myopathy group and grade on the modified Gardner-Medwin and Walton (GMW) grade, color coded as indicated in the legend on the left.

ness in adolescence, and quadriceps weakness by around the age of 30 years. Additionally, patients with ADSSL1 myopathy showed facial weakness and mild CK elevation. ${ }^{2}$

Our study showed that muscle strength was correlated with the degree of fatty replacement in the knee flexors, ankle dorsiflexors, and ankle plantar flexors. This result is compatible with those of previous studies. ${ }^{5,13}$ The muscle strength of the quadriceps was not significantly correlated with the radiological findings, which was probably due to the distal myopathy predominantly affecting distal muscles. Indeed, the participants in the present study exhibited motor weakness of the knee extensors that was milder than that in the knee flexors, ankle dorsiflexors, and ankle plantar flexors. T1-weighted MR images showed characteristic fatty replacement as follows: 1) the early fatty replacement of the tibialis anterior and the short head of the biceps femoris with relatively sparing of the vastus lateralis in GNE myopathy, 2) the early involvement of the posterior compartment of the calf and thigh muscles in dysferlinopathy, and 3) predominant fatty replacement of the gastrocnemius, biceps femoris, and vastus lateralis in ADSSL 1 myopathy. These patterns of fatty replacement in Korean patients with distal myopathy were similar to those found in previous studies of patients with GNE myopathy, dysferlinopathy, and ADSSL1 myopathy. 2,13,14 However, we could not categorize patients with distal myopathy according to their specific causative 


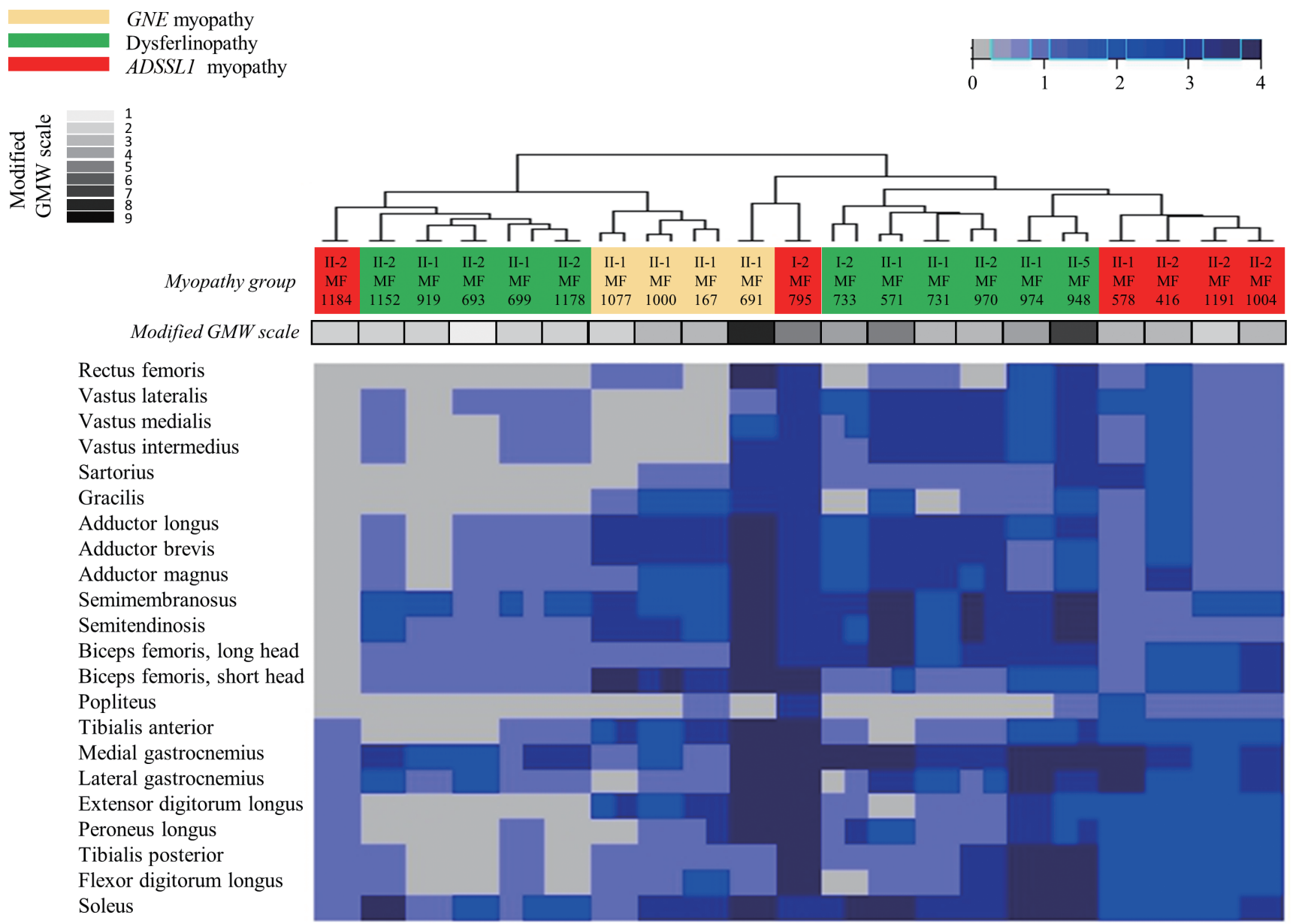

Fig. 3. Hierarchically clustered heat map of Mercuri scale ratings from magnetic resonance (MR) images from 21 patients with distal myopathy. Hierarchical clustering of the Mercuri scale ratings showed that the included patients could be divided into two distinct subpopulations: one with mild fatty replacement and the other with moderate/severe fatty replacement. The main factors contributing to this dichotomy were the causative gene and the clinical severity (as assessed using the modified GMW grade). Each column corresponds to one patient, and they are hierarchically clustered (dendrogram at the top) based solely on MR imaging data (using the Mercuri scale). Each row in the heat map corresponds to one muscle, in descending order from cranial to caudal. A gray-blue-midnight-blue gradient in the heat map indicates increasing fatty substitution (legend at the top). The two rows at the left of the heat map denote individual clinical features (not used in the hierarchical clustering algorithm): the myopathy group and grade on the modified GMW grade, color coded as indicated in the legend on the left. GMW, Gardner-Medwin and Walton.

genes based on the fatty replacement patterns of affected muscles. Thus, we suspect that both the causative genes and the clinical severity impact muscle degeneration.

The present study had two main limitations: 1) it involved only a small number of patients with distal myopathy, and 2) we evaluated only the lower limbs, and not the upper limbs, trunk, or head. This was therefore a pilot study, and so further studies are needed to confirm our results in larger cohorts.

We have identified the characteristic findings in lower-limb MR images of Korean patients with GNE myopathy, dysferlinopathy, and ADSSL1 myopathy. We found that the fatty replacement of muscles in lower-limb MR images was affected by the causative genes and the clinical severity. The present study is the first to reveal the usefulness of lower-limb MR images in the differential diagnosis of distal myopathy in Korea.

\section{Acknowledgements}

We would like to thank the patients who participated in this study and provided their tissues and/or blood for medical research. This study was supported by 2016 academic re- 
search funds of the Korean Society of Clinical Neurophysiology.

\section{Conflicts of Interest}

The scientific significance and materials of our report have not been published previously and will not be submitted for publication elsewhere. The listed authors have no conflicts of interest to declare.

\section{REFERENCES}

1. Bonne G, Rivier F, Hamroun D. The 2018 version of the gene table of monogenic neuromuscular disorders (nuclear genome). Neuromuscul Disord 2017;27:1152-1183.

2. Park HJ, Shin HY, Kim S, Kim SH, Lee Y, Lee JH, et al. Distal myopathy with ADSSL1 mutations in Korean patients. Neuromuscul Disord 2017;27:465-472.

3. Straub V, Carlier PG, Mercuri E. TREAT-NMD workshop: pattern recognition in genetic muscle diseases using muscle MRI: 25-26 February 2011, Rome, Italy. Neuromuscul Disord 2012;22 Suppl 2:S42-S53.

4. Díaz-Manera J, Llauger J, Gallardo E, Illa I. Muscle MRI in muscular dystrophies. Acta Myol 2015;34:95-108.

5. Kinali M, Arechavala-Gomeza V, Cirak S, Glover A, Guglieri M, Feng $L$, et al. Muscle histology vs MRI in Duchenne muscular dystrophy. Neurology 2011;76:346-353.

6. Fanin M, Angelini C. Muscle pathology in dysferlin deficiency.
Neuropathol Appl Neurobiol 2002;28:461-470.

7. Fischer D, Clemen CS, Olivé M, Ferrer I, Goudeau B, Roth U, et al. Different early pathogenesis in myotilinopathy compared to primary desminopathy. Neuromuscul Disord 2006;16:361-367.

8. Jungbluth H, Davis MR, Müller C, Counsell S, Allsop J, Chattopadhyay $A$, et al. Magnetic resonance imaging of muscle in congenital myopathies associated with RYR1 mutations. Neuromuscul Disord 2004;14:785-790.

9. Eisenberg I, Avidan N, Potikha T, Hochner H, Chen M, Olender T, et al. The UDP-N-acetylglucosamine 2-epimerase/N-acetylmannosamine kinase gene is mutated in recessive hereditary inclusion body myopathy. Nat Genet 2001;29:83-87.

10. Sim JE, Park HJ, Shin HY, Nam TS, Kim SM, Choi YC. Clinical characteristics and molecular genetic analysis of Korean patients with GNE myopathy. Yonsei Med J 2013;54:578-582.

11. Liu J, Aoki M, Illa I, Wu C, Fardeau M, Angelini C, et al. Dysferlin, a novel skeletal muscle gene, is mutated in Miyoshi myopathy and limb girdle muscular dystrophy. Nat Genet 1998;20:31-36.

12. Park HJ, Hong JM, Suh GI, Shin HY, Kim SM, Sunwoo IN, et al. Heterogeneous characteristics of Korean patients with dysferlinopathy. J Korean Med Sci 2012;27:423-429.

13. Paradas C, Llauger J, Diaz-Manera J, Rojas-García R, De Luna N, Iturriaga $C$, et al. Redefining dysferlinopathy phenotypes based on clinical findings and muscle imaging studies. Neurology 2010;75:316-323.

14. Tasca G, Ricci E, Monforte M, Laschena F, Ottaviani P, Rodolico $C$, et al. Muscle imaging findings in GNE myopathy. J Neurol 2012;259:1358-1365. 